\title{
Endomyocardial biopsy in diagnosis of cardiomyopathies
}

\author{
K. Somers, ${ }^{1}$ M. S. R. Hutt, ${ }^{2}$ A. K. Patel, ${ }^{3}$ and P. G. D'Arbela \\ From the Department of Cardiology and Department of Pathology, \\ Makerere Medical School, Kampala, Uganda
}

The Konno bioptome, adapted to the intracardiac catheter, has been used in 64 patients in the differential diagnosis of cardiomyopathies, as an additional diagnostic method during standard right heart catheterization and angiocardiography. In the specific instance of endomyocardial fibrosis the Konno biopsy enables histological diagnosis in life. Not surprisingly, in advanced cases dense collagen scar is the usual feature in endomyocardial fibrosis. The Konno method is a real advance in the evaluation and differential diagnosis of heart disease of obscure origin. The technique of biopsy has proved easy to acquire and it is without hazard. Illustrative data from 5 cases of endomyocardial fibrosis and 2 cases of 'congestive cardiomyopathy' are detailed.

Though considerable progress has been achieved in the diagnosis of heart diseases, there remains a group of heart diseases primarily affecting the myocardium the nature of which is obscure, and in which diagnosis is not easily reached in life. In such cases there is usually no obvious antecedent illness or associated lesion. The patients present with acute or chronic cardiac failure and considerable cardiac enlargement may be present. A diversity of conditions occurs in this group of myocardial diseases generally labelled 'cardiomyopathy' and defined by Goodwin (I964) as, 'an acute, subacute or chronic disorder of the heart muscle of unknown or obscure aetiology, often with associated endocardial or sometimes with pericardial involvement but not atherosclerotic in origin'. Increasing awareness of the occurrence of the cardiomyopathies, clinical recognition of primary myocardial disease, and experience at necropsy in various centres in the world indicate an international prevalence (Fejfar, 1968). The exact histopathological lesion in the myocardium, if determined at all, is usually not ascertained before necropsy examination. A biopsy of the myocardium

Received 7 December 1970.

1 Correspondence and requests for reprints to Professor K. Somers, Makerere University Medical School, P.O. Box 7072, Kampala, Uganda.

2 Present address: Department of Morbid Anatomy, St. Thomas' Hospital, London S.E.I.

3 Present address: Veterans Administration Hospital, 2500 Overlook Terrace, Madison, Wisconsin 53705, U.S.A. during life, with careful histological and histochemical studies, offers prospects of information on pathogenesis and pathology and perhaps a long-term possibility of treatment and prevention in areas where the cardiomyopathies are common.

Hitherto, procedures for obtaining biopsy specimens of the myocardium have involved thoracotomy and pericardiotomy or the use of percutaneous needle biopsy techniques. Weinberg, Fell, and Lynfield (1958) used a small thoracotomy incision carried out under local anaesthesia for myocardial and pericardial biopsy in the elucidation of aetiological diagnosis in cases of myocarditis and pericarditis. The need to obviate a surgical incision led to the development of needle techniques in the diagnosis of cardiac pathology. Sutton and Sutton (1960) devised a percutaneous needle technique using a thinwalled, modified Silverman needle with a notched cutting tip. By preference the site used was that of percutaneous left ventricular catheterization (Brock, Milstein, and Ross, 1956). A few years later Soubihe (1963) introduced a needle with a non-cutting tip. After penetration of the heart a lateral window allowed trapping of tissue, subsequently cut with an inner trocar. A needle with a similar principle of entrapment and cutting was widely used by Décourt and his colleagues (1963) in Brazil. Success with needle techniques confirmed the value of the information obtained from histological studies in primary myocardial disorders. However, the amount of 
tissue obtainable by percutaneous needle biopsy is small, and the sample is very localized and ordinarily does not contain endocardium. To meet these challenges Konno developed an instrument, the Konno bioptome, ${ }^{1}$ adapted to the intracardiac catheter, which ensured biopsy material from both endocardium and the myocardium and in larger amounts than obtained from needle biopsy. Konno's encouraging results (Konno and Sakakibara, 1963; Sekiguchi and Konno, I969) led us to explore the usefulness of his technique in the diagnosis of the cardiomyopathies in Uganda with special reference to endomyocardial fibrosis.

\section{Subjects}

In Ugandan experience there are 2 major groups of cardiomyopathy, endomyocardial fibrosis and the 'congestive cardiomyopathies' ('idiopathic cardiomegaly') (Table I). Differential diagnosis between them and rheumatic heart disease can often be difficult, especially when associated with atrioventricular valvular incompetence.

Endomyocardial fibrosis is characterized by mural endocardial lesions in 3 major sites, the apex of the right ventricle, the apex of the left ventricle, and the posterior wall of the left ventricle including the posterior cusp of the mitral valve. At necropsy, biventricular disease is present in 50 per cent of cases though often the lesion on one side is more severe (Edington and Hutt, 1968). The clinical picture is dominated by severe cardiac failure (Somers and Fowler, r968; Somers, Brenton, and Sood, I968b), and differential diagnosis from constrictive pericarditis, a disorder amenable to operation, can often be difficult (Somers et al., I968a, b). Studies of the histopathology of endomyocardial fibrosis suggest that the lesion starts in the connective tissues of the endocardium and adjacent myocardium. This leads to alteration of the overlying endothelium with deposition of platelets and fibrin and subsequent organization. In the later stages, the involved areas are transformed into hard white

TABLE I Incidence of cardiac disorders Cardiac Clinic, Mulago Hospital 1963 to 1969

\begin{tabular}{lrc}
\hline & No. & Per cent \\
\hline Rheumatic heart disease & 524 & $31 \cdot 8$ \\
'Congestive cardiomyopathy' & 201 & $12 \cdot 2$ \\
Endomyocardial fibrosis & 196 & $11 \cdot 8$ \\
Aortitis & 72 & $4 \cdot 4$ \\
Pericardial disease & 50 & $3 \cdot 0$ \\
'Atherosclerotic heart disease' & 45 & $2 \cdot 7$ \\
Cor pulmonale & 36 & $2 \cdot 2$ \\
Congenital heart disease & 349 & $21 \cdot 2$ \\
Miscellaneous/unclassified & 177 & $10 \cdot 7$ \\
\hline Total & I,650 & 100
\end{tabular}

${ }^{1}$ Supplied by Tokyo Hospital Supply Inc., C.P.O. Box 1997, 'Tokyo, Japan. scars composed of collagen and elastica. Characteristic foci of collagen necrosis may be found in the scar tissue. The macroscopical and microscopical appearances of the heart in endomyocardial fibrosis indicate that it is a distinct pathological entity which can be distinguished from other forms of cardiomyopathy (Connor et al., 1967, 1968). Clinical suspicion of endomyocardial fibrosis can be corroborated at cardiac catheterization from analysis of pressure tracings and angiocardiograms (Somers, D'Arbela, and Patel, 197I). In the routine catheter investigation of endomyocardial fibrosis we have used the Konno bioptome as an additional diagnostic method and in staging the disease.

As for the 'congestive cardiomyopathies' ('idiopathic cardiomegaly'), these form a distinct group with considerable cardiac enlargement, presenting in cardiac failure, and unassociated with any special diagnostic features. Mitral and tricuspid incompetence may be present. Response to treatment of the cardiac failure is usually unsatisfactory. At necropsy, there is bilateral ventricular dilatation and hypertrophy, and the heart usually weighs over $400 \mathrm{~g}$, occasionally as much as $700 \mathrm{~g}$. On microscopical examination the myofibres show hypertrophy and there is a variable increase in the connective tissue between the fibrils. The muscle fibres vary considerably in size and often have large nuclei (Edington and Hutt, I968). We have also used Konno's technique of biopsy for evaluating diagnosis in this group of cardiomyopathies and in differentiation from endomyocardial fibrosis.

\section{Konno biopsy method}

For very young children and the occasional apprehensive patient, basal sedation was achieved with diazepam ('Valium') $5 \mathrm{mg}$ orally or by intravenous injection, repeated if required. No other premedication was used in any patient. The right saphenous vein was exposed under local anaesthesia for standard right heart catheterization. Retrograde left heart catheterization was carried out via the right femoral artery. The Konno bioptome (Fig. Ia and b), previously sterilized in the folded position fixed with a tape, was introduced and advanced under fluoroscopic control to the right ventricle (Fig. 2). The bioptome was gently advanced to a point of contact with the ventricular wall which was recognized by a sensation of resistance. At this stage connexion with an exploring electrode, using a sterilized clamp connexion attached to the proximal end of the bioptome, ensured an intracardiac electrocardiographic record indicating chamber position and also contact with the ventricular wall (Emslie-Smith and Somers, I968). Still under fluoroscopic control, the screw at the operator's end controlling the biting mouth of the bioptome was loosened and while pressure was still maintained at the contact area, the now opened biting end was brought to close and the screw tightened again (Fig. Ib). At this stage there may be one or two ventricular ectopic beats 


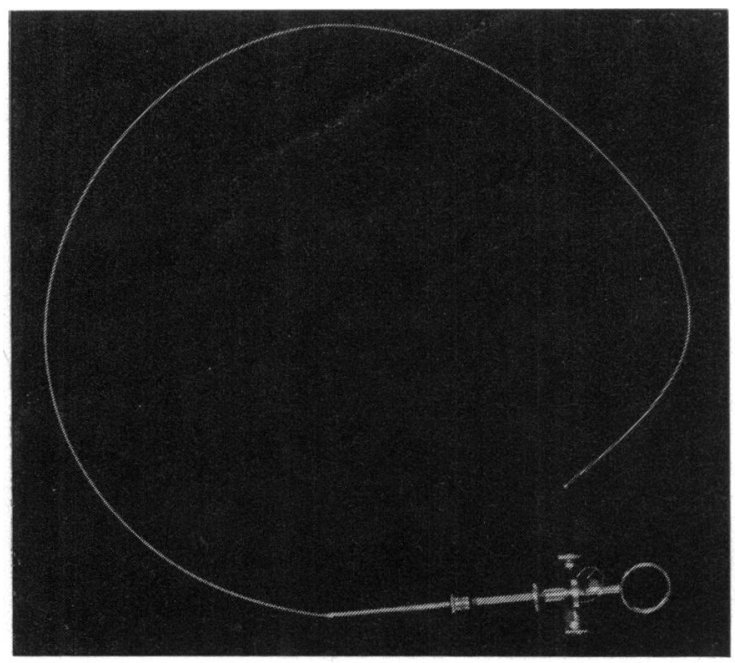

(a)
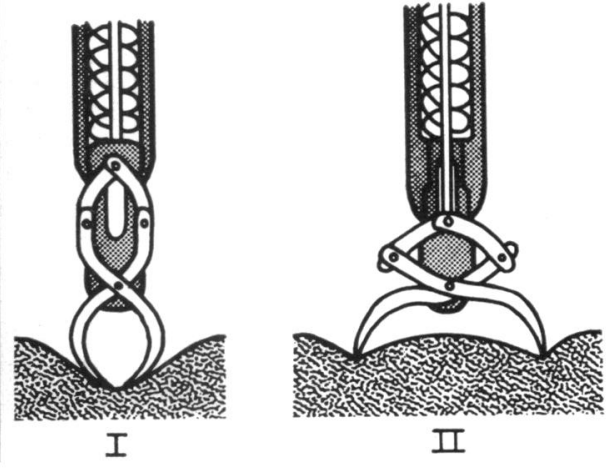

(b)

FIG. I (a) The Konno endomyocardial bioptome. (b) Diagrams showing the action of the Konno bioptome in obtaining endocardial and myocardial biopsy.

(Fig. 3a, b) but this was unusual. No other reaction was noted. The diagnostic investigation was completed by replacement of the bioptome with an N.I.H. catheter and rapid sequence $70 \mathrm{~mm}$ or $16 \mathrm{~mm}$ cinefilm angiocardiography using Cardio-'Conray' contrast medium (Somers et al., 1968c). On removal, the Konno bioptome tip was reopened and a fragment of tissue was removed and suspended in 10 per cent formol saline for subsequent mounting, sectioning, and

FIG. 2 Frame from $70 \mathrm{~mm}$ rapid sequence film showing the Konno bioptome in the cavity of the right ventricle.

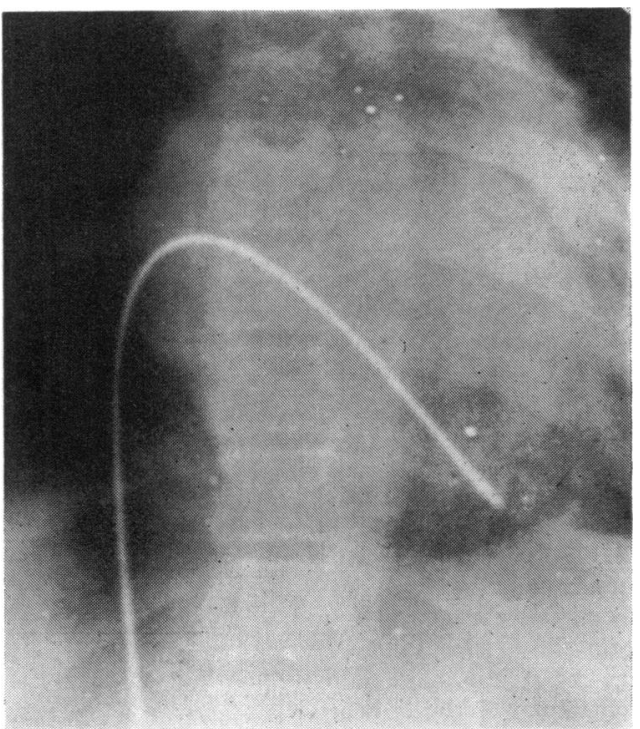

staining. If the initial result was unsuccessful or only blood clot removed, the procedure was repeated with safety as often as four times in our experience with no untoward reaction whatsoever.

In all, 64 patients were biopsied. Their diagnoses are indicated in Table 2. Initially, in this series the success rate with the technique was poor, partly because of excessive caution and partly from inexperience. With experience, the total success rate with single and multiple biopsies approached 80 per cent. Most of the failures occurred in patients with endomyocardial fibrosis where the bioptome tends to slide over the smooth fibrous endocardium. Whenever a biopsy was obtained the findings were usually helpful in establishing the diagnosis. Of the 64 patients submitted to biopsy, 6 were followed through to necropsy. One patient (Case 5) died 2 days later of pulmonary oedema. The remaining 5 patients died after periods ranging from 10 weeks to 4 years. In no case was there any evidence at necropsy of a recognizable site where the biopsy had been taken.

TABLE 2 Diagnosis in 64 patients studied with intracardiac biopsy

\begin{tabular}{lr}
\hline Diagnosis & No. \\
\hline $\left.\begin{array}{lr}\text { Endomyocardial fibrosis } & \\
\quad \text { Biventricular } & 27 \\
\text { Predominant right ventricular } & 16 \\
\quad \text { Predominant left ventricular } & 6\end{array}\right)$ & 49 \\
'Congestive cardiomyopathy' & \\
$\quad$ ('idiopathic cardiomegaly') & 4 \\
Rheumatic heart disease & 6 \\
Constrictive pericarditis & I \\
Unknown & 4 \\
\hline Total & 64 \\
\hline
\end{tabular}

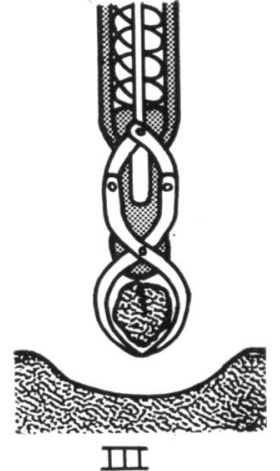

III 
Slides made from blocks of biopsy tissue were stained with haematoxylin and eosin, trichrome, and phosphotungstic acid haematoxylin. In some cases additional stains were used; these included colloidal iron-PAS and Movat's pentachrome methods.

\section{Illustrative case reports}

Case I A r6-year-old Hangaza boy was admitted with a month's history of abdominal swelling. Physical examination revealed jugular venous pressure rise ro $\mathrm{cm}$ above the sternal angle and hepatomegaly of $5 \mathrm{~cm}$. Facial oedema and ascites were present. There was no oedema of the lower limbs. The heart sounds were distant. The blood pressure was $90 / 60 \mathrm{mmHg}$, with a palpable pulsus paradoxus. The electrocardiogram showed a low voltage tracing with $T$ wave flattening throughout. A chest film showed an enlarged rounded heart shadow. A diagnosis of pericardial effusion was confirmed by the aspiration of $500 \mathrm{ml}$ strawcoloured fluid at exploratory pericardiocentesis. The pericardial fluid contained $4 \mathrm{~g}$ protein/100 ml, the microscopical deposit showed lymphocytes, histiocytes, and mesothelial cells. A search for acid-fast organisms in the pericardial fluid was negative. The Heaf test was negative. Blood films showed an eosinophilia of 23 per cent in a WBC of $5000 / \mathrm{mm}^{3}$ Ova of hookworm and Trichiuris trichiura were found in stool specimens. Liver function tests were in the normal range.

Findings at cardiac catheterization are set out in Table 3.

Angiocardiography showed right atrial enlargement and gross tricuspid incompetence. The apical region of the right ventricle was flattened. A moderate pericardial effusion was present. The conclusion after cardiac catheterization was severe right ventricular endomyocardial fibrosis and tricuspid incompetence with associated pericardial effusion. Endomyocardial biopsy was taken from the apical region of the right ventricle.

The microscopical sections (Fig. 4a, b, and c) showed 3 zones: an amorphous thrombus of the type sometimes seen in endomyocardial fibrosis which often appears green at necropsy due to the absence of red cells, a zone of organization with proliferation of capillaries and fibroblasts, and a zone of denser collagen constituting a scar.

This case illustrates various stages seen in the microscopical picture during the development of

\section{TABLE 3 Catheterization data in Case I}

\begin{tabular}{|c|c|c|}
\hline & $m m H g$ & Mean \\
\hline Pulmonary capillary wedge & a I3 v I5 & I4 \\
\hline $\begin{array}{l}\text { Pulmonary artery } \\
\text { Right ventricle }\end{array}$ & $\begin{array}{l}x \underset{y}{x} \text { y } \\
25 / 14 \\
30 / 20\end{array}$ & 17 \\
\hline Right atrium & a $27 \quad$ v 29 & 22 \\
\hline $\begin{array}{l}\text { Left ventricle } \\
\text { Aorta }\end{array}$ & $\begin{array}{l}x 17 \text { y } 17 \\
115 / 12 \\
120 / 85\end{array}$ & \\
\hline
\end{tabular}

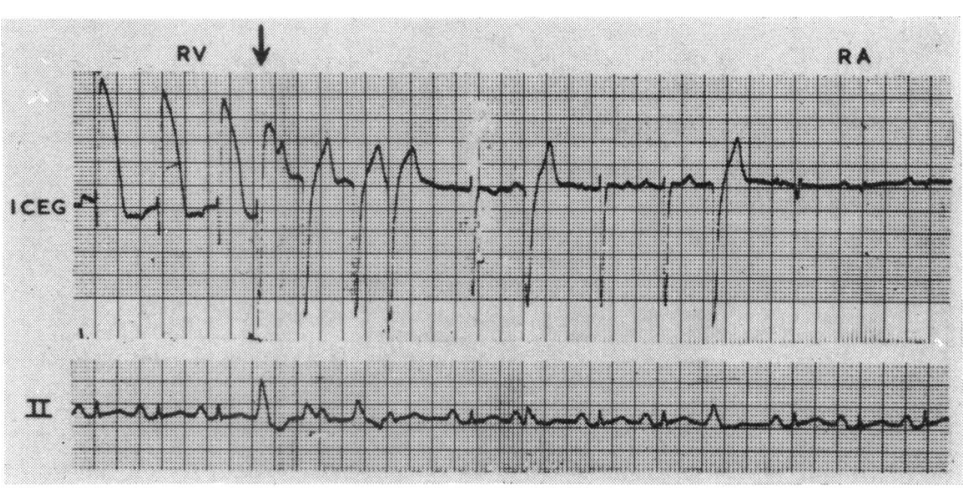

(a)

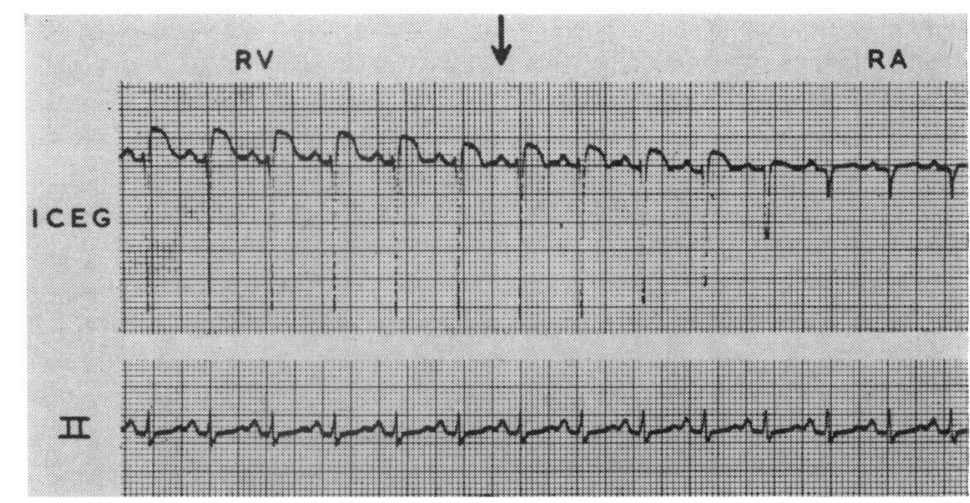

(b)

FIG. 3a Intracardiac electrocardiogram (ICEG) recorded from the right ventricle $(R V)$ using the Konno bioptome as an exploring electrode. ST segment elevation on contact with the endocardium returns to the isoelectric line on withdrawal into the cavity of the right ventricle after the biopsy is taken (arrow). A few ventricular ectopic beats were recorded at the time of biopsy. The closed bioptome was withdrawn into the right atrium (RA).

FIG. 3b Intracardiac electrocardiogram (ICEG) recorded via the Konno bioptome, right ventricle $(R V)$ through right atrium $(R A)$. Endocardial contact shows minimal $S T$ segment elevation. No electrocardiographic reaction occurred at the time of biopsy (arrow).

endomyocardial fibrosis. These appearances are unlikely to be seen in any other cardiac condition affecting the right heart.

Comment A case of a severe and evidently active process of endomyocardial fibrosis, in various stages, as confirmed by intracardiac biopsy. 

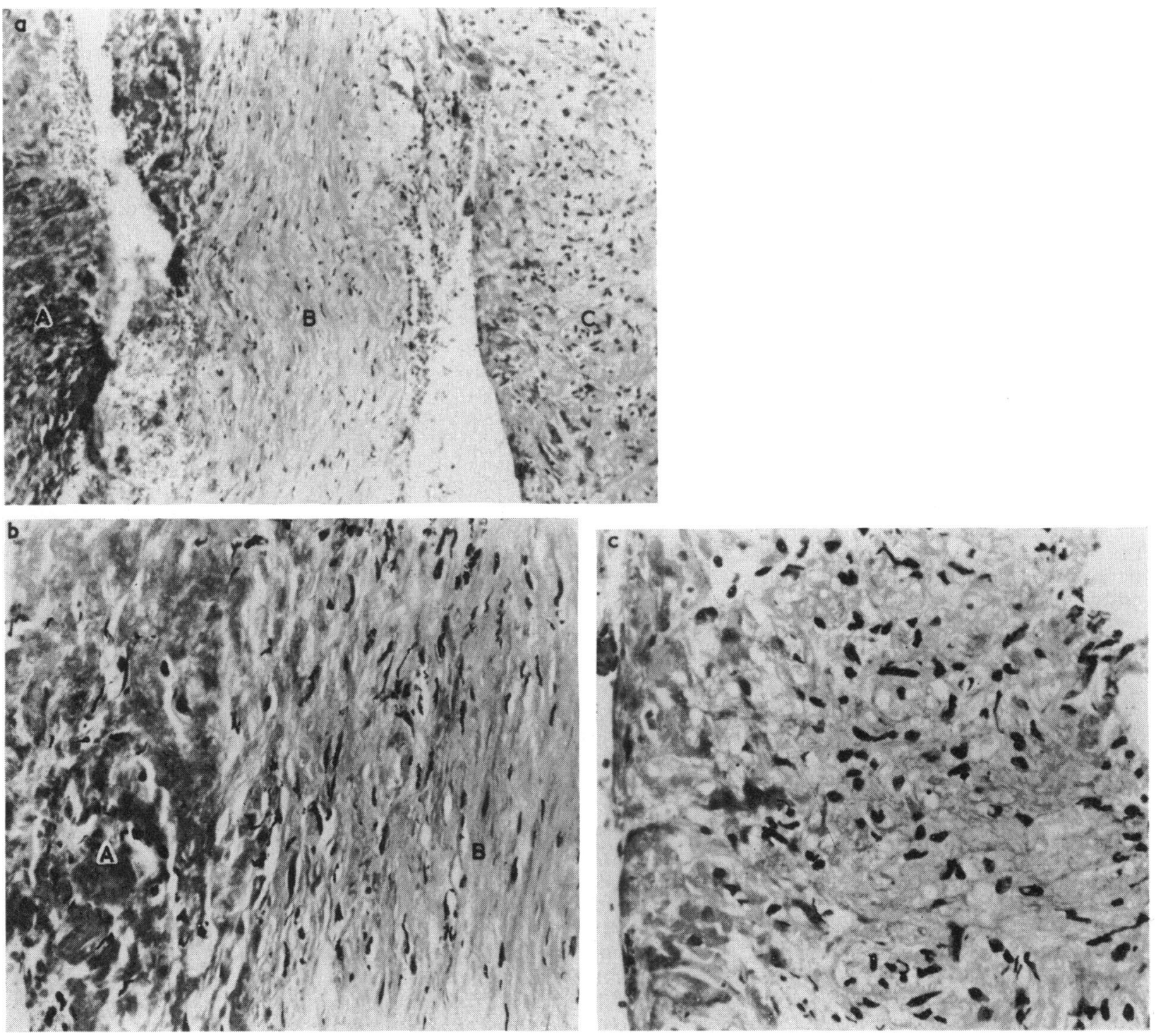

FIG. 4 Case I. (a) Endomyocardial biopsy with 3 layers, on the left a thrombus $(A)$, in the middle $(B)$ a zone of fibrous tissue, and on the right $(C)$, young vascular fibrous tissue.

This appearance is often seen in endomyocardial fibrosis with the young fibrous tissue lying

between the dense scar and the myocardium. (Trichrome $\times$ I0o.) (b) High power view of

junction between newly formed thrombus $(A)$, and endocardial scar $(B)$. (Trichrome $\times 250$.)

(c) Young fibrous tissue with new capillary formation from zone $C$ in $(a)$. (Trichrome $\times 250$.)

Case 2 A 25-year-old female Rwandan patient was referred from a rural hospital with cardiac failure of 4 weeks' duration. She had deteriorated rapidly with choking retrosternal pain, cough, and weakness. She was a para 3 , and the youngest child was 4 years old. On examination, jugular venous pulsation was visible at the ear lobe in the semirecumbent position. The blood pressure was $85 / 70 \mathrm{mmHg}$ and a pulsus paradoxus was noted. The liver was enlarged $4 \mathrm{~cm}$, the spleen
$4 \mathrm{~cm}$, and a small ascites was present. Cardiac dullness was increased to the right, and the apical impulse was unremarkable. A pericardial friction and a third heart sound were heard. The electrocardiogram showed a low voltage tracing with right bundle-branch block and symmetrical $T$ wave inversion in leads VI to V3. The chest film showed a large, rounded heart shadow suggestive of pericardial effusion. Attempted pericardiocentesis yielded no fluid. The Heaf test was 

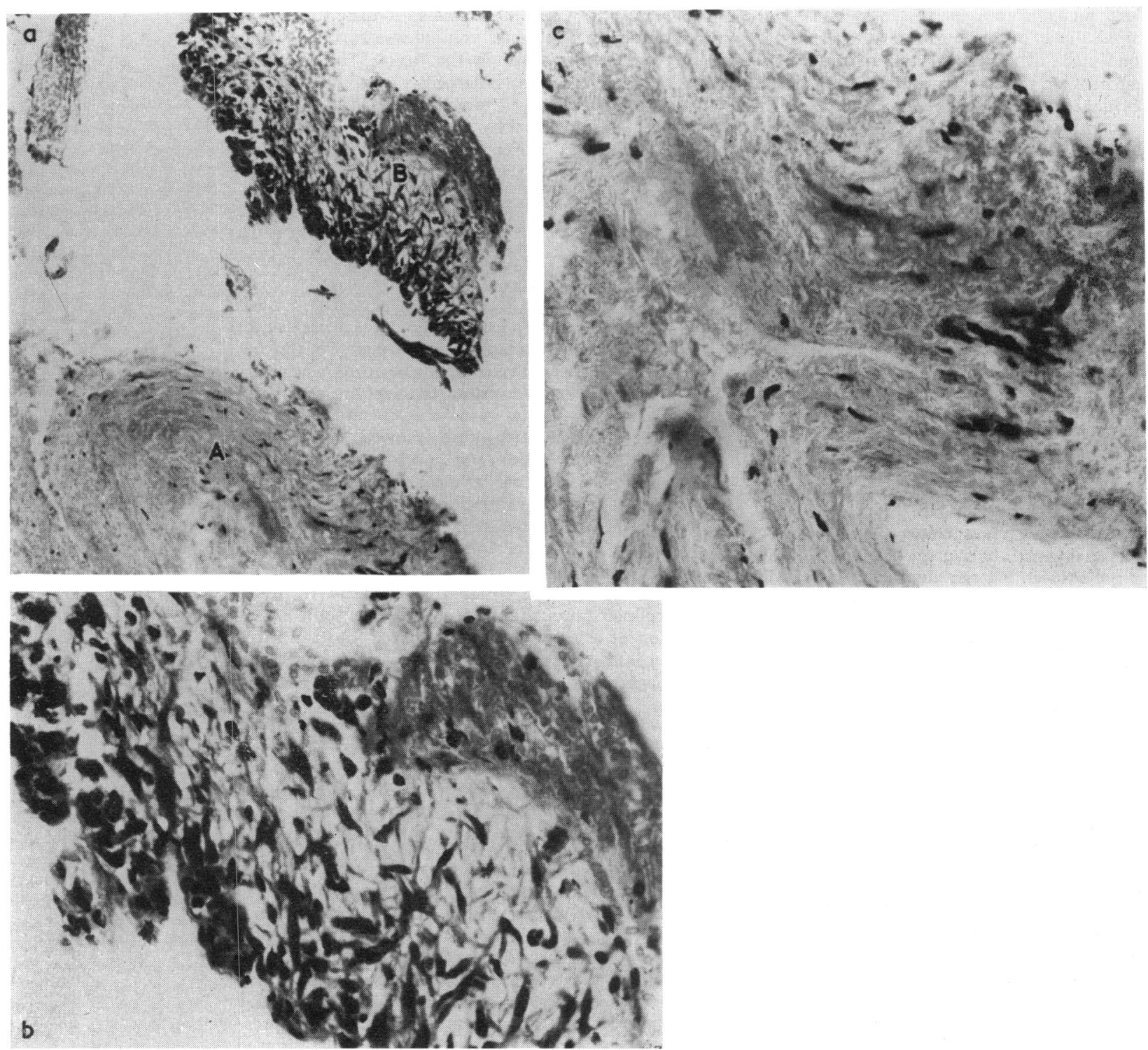

FIG. 5 Case 2. (a) Fragmented biopsy showing a piece of 'mature' fibrous tissue $(A)$ and a separate piece of organizing thrombus $(B)$. (Haematoxylin and eosin $\times 100).(b)$ Organizing thrombus. (Haematoxylin and eosin $\times 250$.) $(c)$ Endocardial scar tissue. (Haematoxylin and eosin $\times 250$.)

negative, antistreptolysin $\mathrm{O}$ titre was 200 Todd units.

Findings at cardiac catheterization are set out in Table 4.

No angiocardiograms were available at this time. Analysis of the pressure records indicated severe right ventricular endomyocardial fibrosis. Biopsy taken 3 times from the endocardium of the right ventricle showed several fragments of tissue. These consisted for the most part of young fibrous tissue containing newly formed capillaries
TABLE 4 Catheterization data in Case 2

\begin{tabular}{|c|c|c|}
\hline & $m m H g$ & Mean \\
\hline Pulmonary capillary wedge & a $18 \quad \mathrm{~V} 12$ & 12 \\
\hline $\begin{array}{l}\text { Pulmonary artery } \\
\text { Right ventricle }\end{array}$ & $\begin{array}{l}x 7 y \quad 9 \\
22 / 12 \\
22 / 8\end{array}$ & 18 \\
\hline Right atrium & a II VI2 & I0 \\
\hline Femoral artery & $\begin{array}{l}x 7 \quad y \quad 7 \\
110 / 75\end{array}$ & \\
\hline
\end{tabular}


and many fibroblasts (Fig. 5a, b, and c). Two small pieces of myocardium were also present and one piece of mature fibrous tissue. The appearances were those of an organizing thrombus with evidence of an older scar and were typical of endomyocardial fibrosis.

Comment The typical fibrous endocardial scar of advanced endomyocardial fibrosis made it difficult to ensure sufficient biopsy material except on 3 attempts. The histological findings of organizing thrombosis and endocardial scar were typical of endomyocardial fibrosis.

Case 3 A 5-year-old Ganda boy was admitted with abdominal swelling for 6 months associated with gross hepatosplenomegaly. Physical examination revealed cyanosis with early finger clubbing, jugular venous engorgement $6 \mathrm{~cm}$ above the sternal angle with brisk $x$ and $y$ descents, and a quiet apical impulse displaced to the anterior axillary line. A gallop rhythm was heard. An electrocardiogram showed tall bifid $\mathbf{P}$ waves, right ventricular preponderance, and ST segment depression in the right chest leads. The chest $x$-ray showed a large globular heart shadow and calcified left hilar lymph nodes. The Heaf test was positive (grade IV).

Findings at cardiac catheterization are set out in Table 5.

The angiocardiogram showed severe apical deformity of the left ventricle. There was no mitral regurgitation. On the right side there was flattening of the right ventricular apex, tricuspid incompetence, and gross right atrial enlargement. The catheter diagnosis was severe biventricular endomyocardial fibrosis.

Endomyocardial biopsy taken from the right ventricle showed 3 pieces of tissue each of which was composed of myocardium and endocardium (Fig. 6a, b). There was extensive fibrosis of the endocardium which extended into the myocardium and led to considerable atrophy and replacement of myofibrils. The appearances were typical of endomyocardial fibrosis.

Comment Endomyocardial scarring and myofibre changes in this patient confirmed the diagnosis of endomyocardial fibrosis.

Case 4 This ro-year-old Rundi girl had a 'fever' followed by facial puffiness and abdominal disten-

TABLE 5 Catheterization data in Case 3

\begin{tabular}{llc}
\hline & $m m H g$ & Mean \\
\hline Pulmonary artery & $20 / 14$ & 17 \\
Right ventricle & $20 / 16$ & \\
Right atrium & a 18 v I8 & 16 \\
\cline { 2 - 3 } & X I4 I4 & \\
Left ventricle & $114 / 1$ & \\
Aorta & $109 / 74$ & \\
Arterial saturation & $60.0 \%$ & \\
\hline
\end{tabular}

sion for 2 weeks. On examination she was deeply cyanosed. The pulse volume was small. A gallop rhythm was heard in the heart. The liver and spleen were both enlarged $8 \mathrm{~cm}$. The electrocardiogram showed low voltage and atrial flutter with varying block. A chest film showed the features of pericardial effusion. Exploratory pericardiocentesis released $400 \mathrm{ml}$ straw-coloured fluid containing $7 \mathrm{~g}$ protein $/ 100 \mathrm{ml}$. Direct examination of the cellular deposit showed a predominance of lymphocytes. Culture of the pericardial fluid failed to isolate Mycobacterium tuberculosis. Serum transaminases were SGOT $52 \mathrm{U}$, SGPT $76 \mathrm{U}$. Repeated pericardiocentesis of volumes up to a litre at a time was necessary for relief of recurrent tamponade. A course of steroids was tried without relief of the pericardial effusion.

Cardiac catheterization findings are set out in Table 6.

A cineangiocardiogram showed considerable compression of the right and left ventricles by a massive pericardial effusion. In addition, the apex of the right ventricle was flattened, suggestive of severe endomyocardial fibrosis.

Biopsy from the right ventricle showed a piece of myocardium and endocardium (Fig. 7a, b). The latter was grossly thickened by relatively acellular fibrous tissue and there was some extension of a looser connective tissue into the myocardium. The appearances were consistent with a diagnosis of endomyocardial fibrosis.

Comment An overall diagnosis of endomyocardial fibrosis was confirmed on the cardiac biopsy findings.

Case 5 A 30-year-old Hangaza woman presented with cough, dyspnoea, and palpitations for 7 months. Examination revealed an orthopnoeic patient with signs of moderately severe congestive cardiac failure. The apical impulse was in the sixth left intercostal space just outside the midclavicular line and quiet in character. A soft early systolic murmur and an opening snap were heard at the mitral area. The pulmonary closure sound was accentuated. The blood pressure was $125 / 95 \mathrm{mmHg}$. A chest $x$-ray showed massive enlargement of the heart with obvious prominence of the pulmonary conus and diffuse mottling in the lungs. The electrocardiogram showed bifid $P$ waves and $T$ wave inversion in the left ventricular leads.

TABLE 6 Catheterization data in Case 4

\begin{tabular}{lll}
\hline & $m m H g$ & Mean \\
\hline Pulmonary capillary wedge & $\frac{\text { v 31 }}{26}$ & 26 \\
Pulmonary artery & $36 / 24$ & 29 \\
Right ventricle & $35 / 22$ & \\
Right atrium & $\frac{\text { v 23 }}{\text { y 21 }}$ & 22 \\
Femoral artery & I09/64 & \\
\hline
\end{tabular}


TABLE 7 Catheterization data in Case 5

\begin{tabular}{lll}
\hline & $m m H g$ & Mean \\
\hline Pulmonary capillary wedge & & 30 \\
$\begin{array}{l}\text { Pulmonary artery } \\
\text { Right ventricle }\end{array}$ & $113 / 44$ & 68 \\
Right atrium & I $6 / 24$ & \\
\cline { 2 - 3 } & a 25 12 y 15 & \\
\hline
\end{tabular}

Cardiac catheterization findings are set out in Table 7.

A left ventricular angiocardiogram showed a 'cupped' apex with a shelf effect at the rim of the cup and a mild mitral incompetence. A right ventricular angiocardiogram showed obvious prominence of the pulmonary artery together with a grossly dilated and hypertrophied right ventricle with exaggerated septal crenations. The right ventricular apex was preserved, and a moderate tricuspid incompetence was present. The conclusion was severe endomyocardial fibrosis of the left ventricle with severe pulmonary hypertension and secondary right heart failure.

Biopsy taken from the right ventricular endocardium showed obvious hypertrophy of the myofibres with some patchy interstitial fibrosis (Fig. 8). The patient died 2 days later in acute pulmonary oedema. At necropsy there was evidence of gross left-sided endomyocardial fibrosis with dilatation and hypertrophy of the right ventricle. One tiny patch of endocardial scarring was seen at the apex of the right ventricle. There was no recognizable evidence anywhere in the right ventricle to indicate the site of biopsy.

Comment Right ventricular myofibre hypertrophy on biopsy alone, while compatible with any situation of pulmonary hypertension, would not be diagnostic of endomyocardial fibrosis. In this instance, 'non-specific' right ventricular hypertrophy was associated with mitral auscultatory findings characteristic of left ventricular endomyocardial fibrosis (Fowler and Somers, 1968). With the whole heart available at necropsy the connective tissue changes in the right ventricular biopsy sections were consistent with pancardiac involvement in endomyocardial fibrosis.

Case 6 This 30-year-old Ganda woman, a para 5 , was a known case of congestive cardiac failure for 3 months. Her symptoms began when her youngest child was 4 months old. Physical examination revealed $8 \mathrm{~cm}$ jugular venous engorgement, massive oedema of the sacrum and lower limbs, and ascites. The apical impulse was in the fifth space, in the anterior axillary line. A gallop rhythm was easily heard. There was a soft tricuspid systolic murmur. The blood pressure was $100 / 80 \mathrm{mmHg}$. The electrocardiogram showed a biphasic $P$ wave in VI and flattening of the $T$ waves in leads aVL and the left ventricular leads. The blood picture was normal. Blood
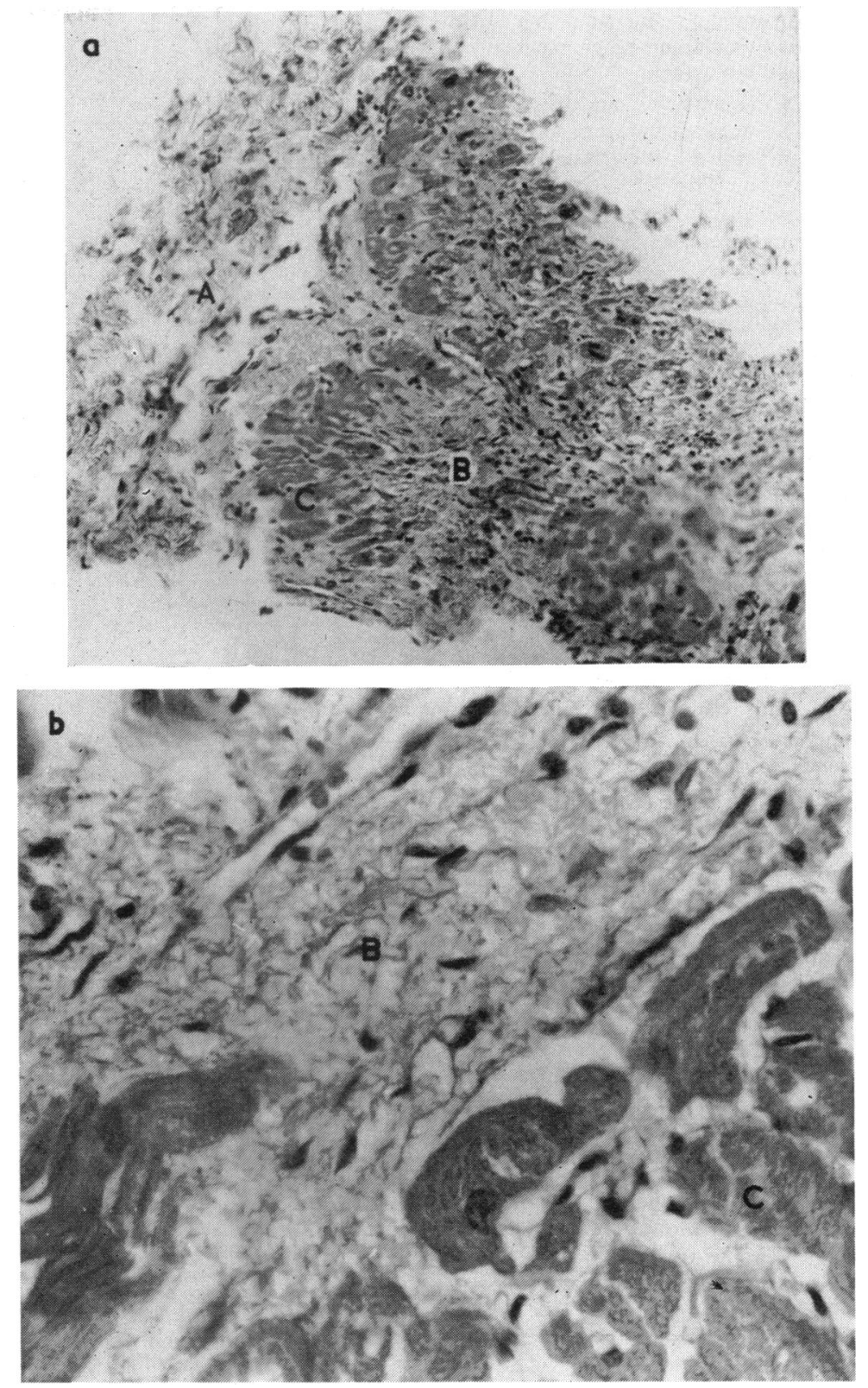

FIG. 6 Case 3. (a) Fibrosis of the endocardium $(A)$ with extension into myocardium (B) with loss of myocardial fibres. There is a zone of surviving myocardium just beneath the endocardium $(C)$. This pattern with deeper myocardial scarring is seen in endomyocardial fibrosis. (Haematoxylin and eosin $\times 100$.)

(b) High power view of myocardial scarring

$(B)$ with preservation of zone beneath endocardium $(C)$. (Haematoxylin and eosin $\times 250$.) 
cultures were negative. $X$-ray of the chest showed a grossly enlarged heart, especially to the left, with a large left atrium.

Cardiac catheterization data are set out in Table 8.

The angiocardiogram showed a free tricuspid incompetence, the right ventricle was grossly enlarged. The left ventricle was also enlarged. Mitral incompetence was noted. The catheter findings were consistent with 'congestive cardiomyopathy'.

Endomyocardial biopsy of the right ventricle revealed gross hypertrophy of the myofibres, many of which had a central vacuolated appearance (Fig. 9a, b). The myofibre nuclei were enlarged and sometimes bizarre in shape. The interstitial tissue did not appear abnormal. The appearances were compatible with the findings in 'idiopathic cardiomegaly'.

Comment Myofibre hypertrophy and vacuolation ('foamy' change) with nuclear enlargement is characteristic of 'congestive cardiomyopathy' ('idiopathic cardiomegaly').

Case 7 This 13-year-old Rundi girl gave a 2-month history, starting with cough, haemoptysis, and dyspnoea followed by ascites and oedema of the lower limbs.

The jugular venous pressure was raised $10 \mathrm{~cm}$ above the sternal angle, the liver was enlarged 3 fingers breadth. Blood pressure was $100 / 80$ mmHg. There was a palpable heave in the left upper parasternum, and the apical impulse was normally located. A soft short apical systolic murmur was heard at the mitral area. A gallop was widely heard. The blood picture showed a moderate anaemia ( $\mathrm{Hb}$ II.2 $\mathrm{g} / 100 \mathrm{ml}$ ) and an eosinophilia of 30 per cent in a total leucocyte count of $7,800 / \mathrm{mm}^{3}$. Stool examination showed hookworm ova. The antistreptolysin $O$ litre was 200 Todd units.

The chest film showed gross cardiac enlargement to the left and to the right, with considerable pulmonary congestive changes. The electrocardiogram showed a low voltage tracing with flat $T$ waves throughout.

Findings at cardiac catheterization are given in Table 9.

Angiocardiography demonstrated mitral and tricuspid incompetence. The outlines of the ventricles appeared normal though dilated. The clinical and catheterization findings were consist-

\section{TABLE 8 Catheterization data in Case 6}

\begin{tabular}{lll}
\hline & $m m H g$ & Mean \\
\hline Pulmonary capillary wedge & a 37 v 58 & 32 \\
\cline { 2 - 3 } ×ulmonary artery & 54 y 32 & \\
Right ventricle & $57 / 35$ & 45 \\
Right atrium & $\frac{\text { a I5 v I2 }}{12}$ & \\
Left ventricle & x 10 y I0 & \\
Aorta & $120 / 35$ & \\
\hline
\end{tabular}
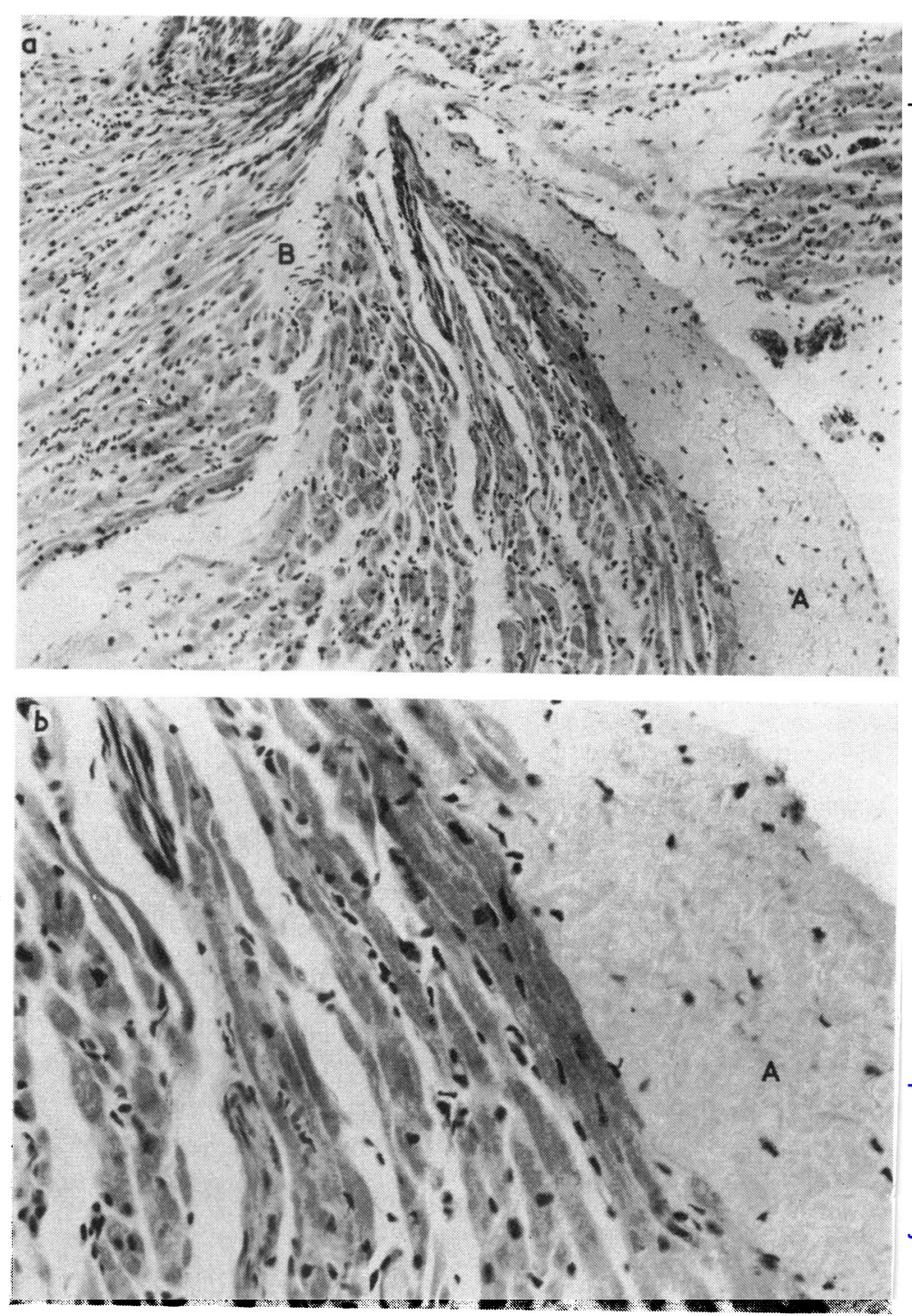

FIG. 7 Case 4. (a) Conspicuous thickening of right ventricular endocardium $(A)$ with some extension of fibrosis in myocardium. (Trichrome $\times$ 100.) (b) Thickened endocardium with mature collagen scar $(A)$. (Trichrome $\times 250$.)

ent with a diagnosis of 'congestive cardiomyopathy'.

Endomyocardial biopsy showed a piece of ventricular muscle with hypertrophy of the myofibres, some of which contained large and rather bizarrely shaped nuclei (Fig. IO). There was a slight increase in interstitial tissue but no evidence of myocarditis.

Comment The biopsy findings in this patient were consistent with a diagnosis of 'congestive cardiomyopathy' ('idiopathic cardiomegaly'). 
TABLE 9 Cardiac catheterization data in Case 7

\begin{tabular}{|c|c|c|}
\hline & $m m H g$ & Mean \\
\hline Pulmonary capillary wedge & a 32 v 45 & 30 \\
\hline $\begin{array}{l}\text { Pulmonary artery } \\
\text { Right ventricle }\end{array}$ & $\begin{array}{l}\times 26 \text { y } 23 \\
54 / 27 \\
50 / 15\end{array}$ & 38 \\
\hline Right atrium & $\begin{array}{l}\text { a } 14 \text { v I I } \\
x 8 \text { y } 9\end{array}$ & II \\
\hline $\begin{array}{l}\text { Left ventricle } \\
\text { Aorta }\end{array}$ & $\begin{array}{l}97 / 29 \\
90 / 67\end{array}$ & \\
\hline
\end{tabular}

\section{Conclusions}

The diagnosis of obscure heart disease (cardiomyopathy) may be difficult even after death when the whole organ is available for study. However, as already indicated, endomyocardial fibrosis is a specific pathological entity (Connor et al., 1967, 1968). Analysis of biopsy material obtained from patients with proved endomyocardial fibrosis shows that it is possible with the Konno biopsy to make a histological diagnosis during life. Not surprisingly, in some cases with dense collagen scars over the right ventricle it is not easily possible to obtain sufficient material.

In the 'congestive cardiomyopathies', in which a successful biopsy is usual, the histological picture of myofibre hypertrophy and nuclear changes is helpful in confirmation of the diagnosis of 'idiopathic cardiomegaly' and in differentiation from endomyocardial fibrosis. It is likely that more conclusive diagnoses can be made by the Konno method in more

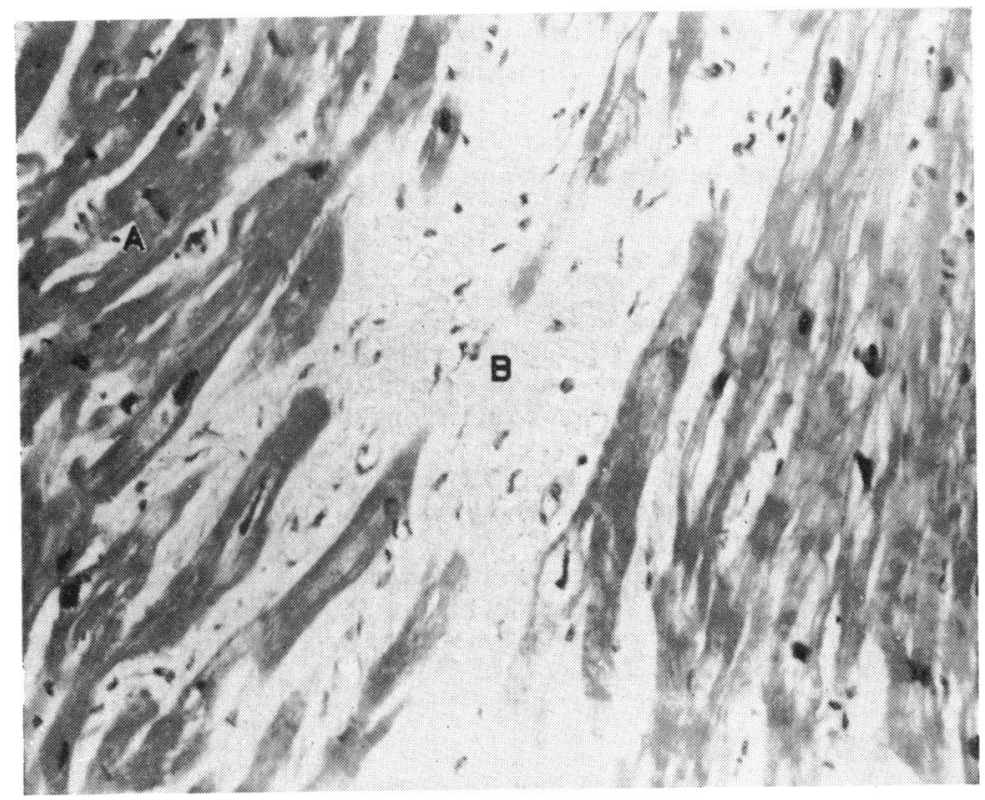

FIG. 8 Case 5. Hypertrophied myofibres in the right ventricle $(A)$ showing some loss of fibres and replacement by collagen (B).

(Haematoxylin and eosin $\times 250$.)

'specific' myocardial disorders with an absolutely characteristic lesion such as amyloidosis, myocarditis, or haemochromatosis. It must be stressed that interpretation of the biopsy findings must be made in the light both of the clinical features and of the cardiac disease pattern in any particular area.

FIG. 9 Case 6. (a) Gross hypertrophy of myofibres with vacuolation in the perinuclear area. $($ Trichrome $\times$ 150.) (b) As (a). (Phosphotungstic acid haematoxylin $\times 150$.)

(a)

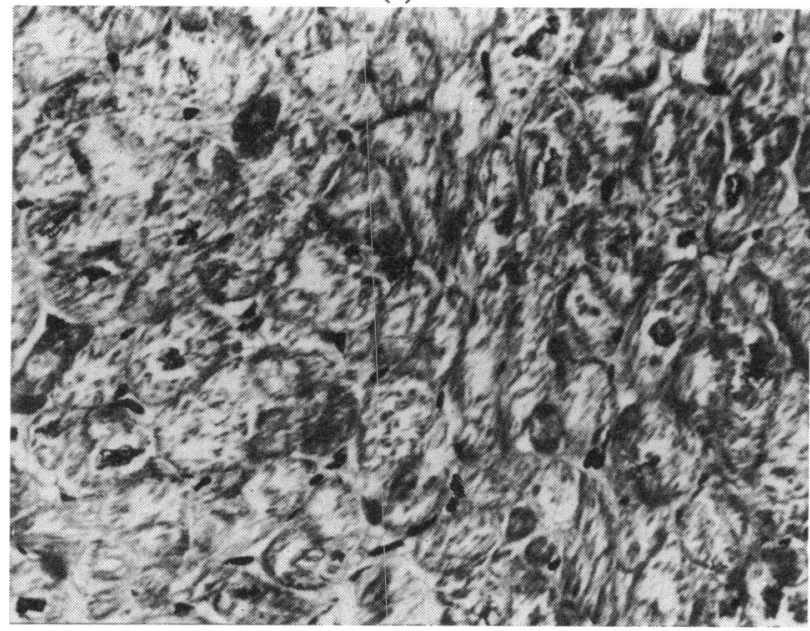

(b)

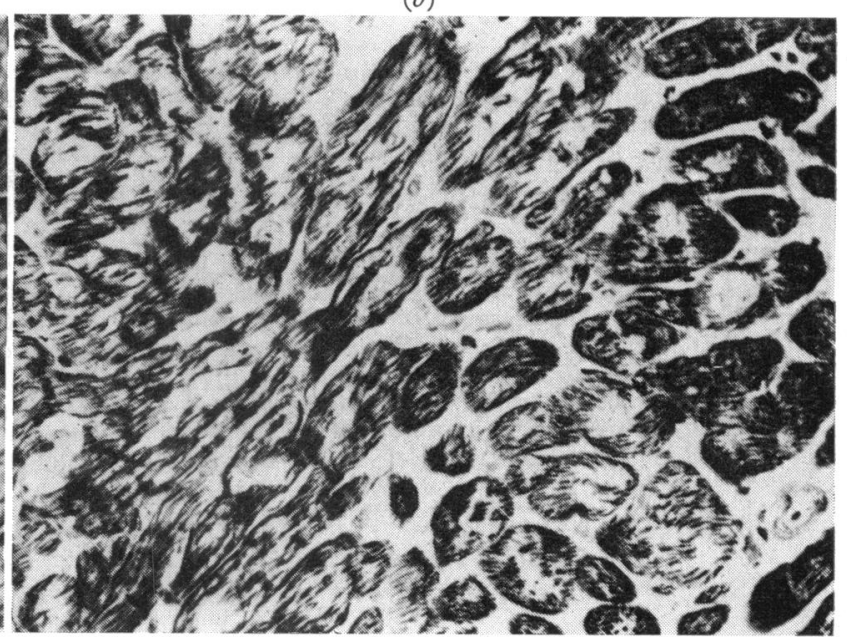


The possibilities of electron microscopical, immunopathological, and virological studies in fresh tissue obtained from patients suffering from the cardiomyopathies may be facilitated by greater availability of the Konno technique of biopsy.

We are grateful to Dr. Souji Konno for the gift of the bioptome and for instruction in its use, and to Mr. John Milton, senior cardiological technician, who demonstrated the value of connecting the bioptome as an exploring electrode in locating the site of biopsy.

The British Heart Foundation and Grindlays Bank, Uganda, have supported one of us (K.S.) generously with research grants.

Diazepam ('Valium') for oral and intravenous use was supplied to us by the kindness of Dr. John Garrod of Roche Products. Cardio-' Conray' was supplied by May and Baker Ltd.

\section{References}

Brock, R., Milstein, B. B., and Ross, D. N. (1956). Percutaneous left ventricular puncture in the assessment of aortic stenosis, Thorax, II, I63.

Connor, D. H., Somers, K., Hutt, M. S. R., Manion, W. C., and D'Arbela, P. G. (I967). Endomyocardial fibrosis in Uganda (Davies' disease), Part I. An epidemiologic, clinical, and pathologic study. American Heart fournal, 74, 687.

Connor, D. H., Somers, K., Hutt, M. S. R., Manion, W. C., and D'Arbela, P. G. (I968). Endomyocardial fibrosis in Uganda (Davies' disease). Part II. American Heart fournal, 75, 107.

Décourt, L. V., Garcia, D. P., Montenegro, M. R. and Tsuzuki, S. (1963). Estudo da patologia do endocárdio por meio da biopsia do coração humano. Revista da Hospital das Clínicas de Faculdade de Medicina da Universidade de São Paulo, 18, 247.

Edington, G. M., and Hutt, M. S. R. (1968). Idiopathic cardiomegaly. General and pathology. Cardiologia, 52, 33.

Emslie-Smith, D., and Somers, K. (1968). Intracardiac electrocardiogram in endomyocardial fibrosis. British Heart fournal, 30, 696.

Fejfar, Z. (1968). Cardiomyopathies - an international problem. Cardiologia, 52, 9.

Fowler, J. M., and Somers, K. (1968). Left-ventricular endomyocardial fibrosis and mitral incompetence. A new syndrome. Lancet, $1,227$.

Goodwin, J. F. (1964). Cardiac function in primary myocardial disorders. British Medical fournal, $\mathbf{r}$, 1527.

Konno, S., and Sakakibara, S. (1963). Endo-myocardial biopsy. Diseases of the Chest, 44, 345 .

Sekiguchi, M., and Konno, S. (1969). Histopathological differentiation employing endomyocardial biopsy in the clinical assessment of primary myocardial disease. Fapanese Heart fournal, 10, 30.

Somers, K., D'Arbela, P. G., and Patel, A. K. (I97I). Endomyocardial fibrosis, a clinical review, in medicine in a tropical environment. British Medical fournal, to be published.

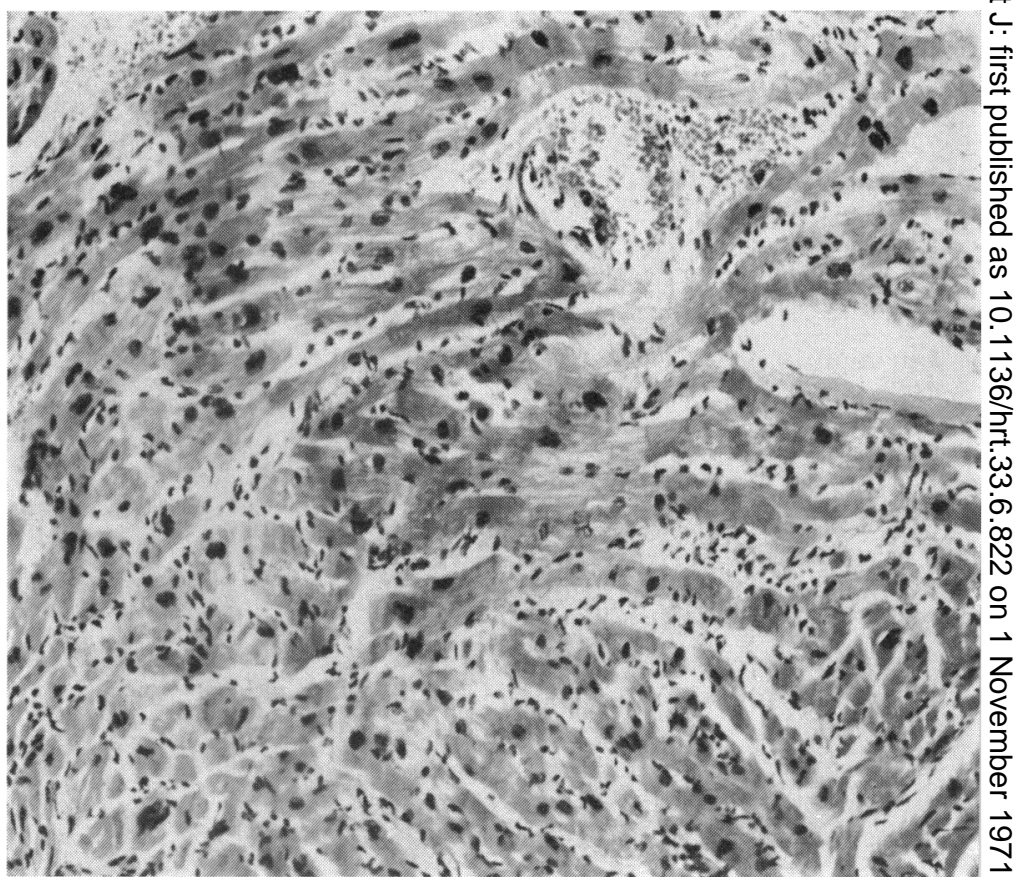

FIG. IO Case 7. Myocardial hypertrophy of right ventricle, with large and variable nuclei. There is some increase in intermyofibrillary tissue and an increase in cells. (Haematoxylin and eosin $\times 128$.)

Somers, K., Brenton, D. P., D'Arbela, P. G., Fowler, J. M., Kanyerezi, B. R., and Sood, N. K. (I968a) Haemodynamic features of severe endomyocardial fibrosis of right ventricle, including comparison with constrictive pericarditis. British Heart fournal, 30, 322.

Somers, K., Brenton, D. P., and Sood, N. K. (1968b). Clinical features of endomyocardial fibrosis of the right ventricle. British Heart fournal, 30, 309.

Somers, K., and Fowler, J. M. (1968). Endomyocardial fibrosis: clinical diagnosis. Cardiologia, 52, 25.

Somers, K., Patel, A. K., Fowler, J. M., and D'Arbela, P. G. (1968c). A clinical trial of Cardio-'Conray' and comparison with 'Conray' 420 in angiocardiography. British fournal of Radiology, 41, 678.

Soubihe, N. V. (1963). Punção-biopsiã do coração. Archivos del Instituto de Cardiología de México, 33, 132.

Sutton, D. C., and Sutton, G. C. (1960). Needle biopsy of the human ventricular myocardium: Review of 54 consecutive cases. American Heart fournal, 60, 364 .

Weinberg, M., Fell, E. H., and Lynfield, J. (1958). Diagnostic biopsy of the pericardium and myocardium. Archives of Surgery, 76, 825 . 